Pacific Journal of Mathematics

TWO CHARACTERIZATIONS OF COMMUTATIVE BAER 


\title{
TWO CHARACTERIZATIONS OF COMMUTATIVE BAER RINGS
}

\author{
JOSEPH KIST
}

\begin{abstract}
A commutative ring $A$ is called a Baer ring if the annihilator of each element in $A$ is the principal ideal generated by an idempotent. It is shown that the following three conditions on a semiprime commutative ring $A$ with identity are equivalent: (1) $A$ is a Baer ring, (2) the mapping $Q \rightarrow Q \cap E$ is a homeomorphism of $\operatorname{Min} \operatorname{Spec} A$ with the Boolean space of the Boolean algebra $E$ of idempotents in $A$, (3) Min Spec $A$ is a retract of $\operatorname{Spec} A$.
\end{abstract}

Introduction. A commutative ring $A$ is called a Baer ring if the annihilator of each element in $A$ is the principal ideal generated by an idempotent. Baer rings have been the subject of several recent investigations. (See, e.g., [1], [2], [8], [9], [10], [14], [15], [16] and [17].) The main purpose of this note is to give two new characterizations of these rings.

All rings considered in this paper are assumed to be commutative with identity; the symbol $A$ will always denote such a ring, and $E=$ $E(A)$ will denote the Boolean algebra of idempotents in $A$. Recall that the operations in $E$ are given by $e \cap f=e f, e^{\prime}=1-e$, and hence $e \cup f=\left(e^{\prime} \cap f^{\prime}\right)^{\prime}=e+f-e f$.

If $\mathscr{V}=\mathscr{V}(A)$ is any family of prime ideals in $A$, and if $\alpha$ is an element of $A$, then let $\mathscr{V}_{a}=\{Q \in \mathscr{V}: a \notin Q\}$. We have $\mathscr{V}_{a} \cap \mathscr{V}_{b}=\mathscr{V}_{a b}$, and $\mathscr{V}_{i}=\mathscr{Y}$, so the family $\left\{\mathscr{V}_{a}: a \in A\right\}$ is a base for a topology on $\mathscr{Y}$; this topology is called the Stone or Zariski topology. It is to be understood that any set of prime ideals carries the Stone-Zariski topology.

The minimal prime spectrum of $A$, denoted by $\mathscr{P}(A)$, or also by Min Spec $A$, is the space of minimal prime ideals of $A$. As shown in [6] and [9], $\mathscr{P}=\mathscr{P}(A)$ is a Hausdorff space in which each set $\mathscr{P}_{a}$ is both open and closed.

The set $\mathscr{P}(E)$ of maximal (= prime) ideals in the Boolean algebra $E$ is topologized by taking the family $\left\{\mathscr{P}_{e}(E): e \in E\right\}$ as a base, where $\mathscr{P}_{e}=\{P \in \mathscr{P}(E): e \notin P\}$. When so topologized, $\mathscr{P}(E)$ is a compact Hausdorff space in which each set $\mathscr{P}_{e}(E)$ is both open and closed; moreover, each open and closed subset of $\mathscr{P}(E)$ is of the form $\mathscr{P}_{e}(E)$ for some $e$ in $E$.

If $Q$ is a prime ideal in $A$, then $Q \cap E$ is a prime ideal in $E$, i.e., it is a member of $\mathscr{P}(E)$. Our first characterization of Baer rings is the following one. (Recall that a semiprime ring is one in which 
there are no nonzero nilpotents.)

THEOREM 1. A semiprime commutative ring $A$ with identity is a Baer ring if and only if the mapping $Q \rightarrow Q \cap E$ is a homeomorphism of $\mathscr{P}(A)$ upon $\mathscr{P}(E)$.

Section 1 of this paper will be devoted to a proof of the above result. In the course of proving it, we show that for any ring $A$, the mapping $Q \rightarrow Q \cap E$ is always a continuous surjection of $\mathscr{P}(A)$ upon $\mathscr{P}(E)$, and we characterize those rings $A$ for which this mapping is a bijection.

REMARKs 1. If $X$ is a nonempty set, and if $Z$ is the ring of integers, then $A=Z^{X}$ is a Baer ring. In [13], D. Scott showed that there is a bijection of the set of minimal prime ideals of $A$ upon the set of ultrafilters on $X$. The Boolean algebra $E(A)$ is isomorphic to the Boolean algebra of all subsets of $X$, and $\mathscr{P}(E)$ is in one-to-one correspondence with the ultrafilters on $X$. Hence, Theorem 1 is a generalization of Scott's result.

2. It was stated without proof in [10] that $\mathscr{P}(A)$ and $\mathscr{P}(E)$ are homeomorphic when $A$ is a Baer ring. However, my original proof of that fact was roundabout, and different from the one given here.

As our second characterization, we show that a semiprime commutative ring $A$ with identity is a Baer ring if and only if $\mathscr{P}(A)$ is a retract of Spec $A$, the space of all prime ideals in $A$. This result, Theorem 2 , is proved in $\S 2$.

Theorems 1 and 2 are applied in $\S 3$ to obtain a new proof of the known result that a semiprime commutative ring $A$ with identity is a regular ring if and only if each prime ideal in $A$ is maximal. In $\S 4$, Theorem 2 is applied to generalize a result of Henriksen and Jerison.

In his 1972 Tulane thesis [Baer rings and their structure sheaves], Howard Evans independently established Theorems 1 and 2, and even for noncommutative Baer rings; his methods of proof are entirely different from the methods we use here.

1. Proof of Theorem 1. Recall that $\operatorname{rad} J$, the radical of an ideal $J$ in a commutative ring $A$, is the set of all elements $a$ in $A$ such that some power of $a$ is in $J$. If $I$ is an ideal in the Boolean algebra $E$, then we denote by $\bar{I}$ the ideal in $A$ generated by $I$. It is easy to see that $\bar{I}$ consists of all elements $a$ such that $a e^{\prime}=0$ for some element $e$ in $I$.

We shall prove Theorem 1 by a sequence of lemmas. 
LEMmA 1.1. If $I$ is an ideal in $E$, then rad $\bar{I}$ is the intersection of all minimal prime ideals containing $I$.

Proof. Let $a \notin \operatorname{rad} \bar{I}$ so that $a^{n} \notin \bar{I}$ for each natural number $n$. Thus, $a^{n} e^{\prime} \neq 0$ for each nonnegative integer $n$ and each element $e$ of $I$. The family $S$ of all such elements is a multiplicative semigroup, for $a^{m} e^{\prime} a^{n} f^{\prime}=a^{m+n}(e \cup f)^{\prime}$, and $e \cup f$ is in $I$ if both $e$ and $f$ are. Since $0 \notin S$, Krull's lemma [7, p. 1] guarantees the existence of a minimal prime ideal $Q$ which does not meet $S$. It follows that $a \notin Q$ and that $I \subset Q$.

CoRollary 1.2. If $P$ is a prime ideal in $E$, then there is a minimal prime ideal $Q$ in $A$ such that $P=Q \cap E$.

Proof. By the lemma, there is a minimal prime ideal $Q$ in $A$ such that $\bar{P} \subset Q$. Thus, $P \subset Q$, and so $P=Q \cap E$.

The previous result asserts that the mapping $Q \rightarrow Q \cap E$ is a surjection of $\mathscr{P}(A)$ upon $\mathscr{P}(E)$. If $e \in E$, then $\{Q \in \mathscr{P}(A): e \notin Q \cap E\}=$ $\{Q \in \mathscr{P}(A): e \notin Q\}$, and the latter set is open (and closed) in $\mathscr{P}(A)$. Hence, for any commutative ring $A$ with identity, the mapping $Q \rightarrow$ $Q \cap E$ is a continuous surjection of $\mathscr{P}(A)$ upon $\mathscr{P}(E)$.

LEMMA 1.3. Let $A$ be a semiprime commutative ring with identity. If $I$ is an ideal in $E$, then the ideal $\bar{I}$ coincides with its radical.

Proof. Let $a^{n} \in \bar{I}$, so that $a^{n} e^{\prime}=0$ for some $e \in I$. Then $\left(a e^{\prime}\right)^{n}=0$, and so $a e^{\prime}=0$, that is, $a \in \bar{I}$.

An ideal $J$ in $A$ is called regular if $J=\overline{J \cap E}$.

Proposition 1.4. In a semiprime commutative ring $A$ with identity, the surjection $Q \rightarrow Q \cap E$ of $\mathscr{P}(A)$ upon $\mathscr{P}(E)$ is a bijection if and only if each minimal prime ideal in $A$ is regular.

Proof. Suppose that each minimal prime ideal in $A$ is regular. If $Q_{1}$ and $Q_{2}$ are minimal primes such that $Q_{1} \cap E=Q_{2} \cap E$, then $Q_{1}=$ $\overline{Q_{1} \cap E}=\overline{Q_{2} \cap E}=Q_{2}$, and thus the mapping $Q \rightarrow Q \cap E$ is an injection.

Conversely, suppose there is a nonregular minimal prime ideal $Q$ in $A$. Choose $a$ in $Q$ such that $a \notin \overline{Q \cap E}$. By Lemmas 1.1 and 1.3, there is a minimal prime ideal $Q_{1}$ such that $a \notin Q_{1}$, and $Q \cap E \subset Q_{1}$. Thus, $Q \cap E=Q_{1} \cap E$ but $Q \neq Q_{1}$.

Let $A$ be a ring in which the mapping $Q \rightarrow Q \cap E$ is a bijection of $\mathscr{P}(A)$ upon $\mathscr{P}(E)$. If $P \in \mathscr{P}(E)$ then there is exactly one element 
$Q \in \mathscr{P}(A)$ for which $P=Q \cap E$, and hence $\operatorname{rad} \bar{P}=Q$. Thus, if $A$ is semiprime, then $\bar{P}=Q$, and consequently the inverse of the above mapping is $P \rightarrow \bar{P}$.

If $a$ is an element of a commutative ring $A$, then ann $a$, the annihilator of $a$, is the set of all elements $b$ in $A$ for which $a b=0$. The following characterization of minimal prime ideals can be found in [9]; see also [7, p. 57].

LEMMA 1.5. A prime ideal $Q$ in a semiprime commutative ring is a minimal prime ideal if and only if ann $a \not \subset Q$ whenever $a \in Q$.

Lemma 1.6. Each minimal prime ideal in a Baer ring is regular.

Proof. Let $Q$ be a minimal prime ideal in the Baer ring $A$, and let $a$ be an element of $Q$. There is an idempotent $e$ such that ann $a=A e$. A Baer ring is semiprime [9], so Lemma 1.5 insures that $e \notin Q$. Hence, $a \in \overline{Q \cap E}$, and therefore $Q=\overline{Q \cap E}$.

The following result can be found in [6] and [9].

LEMMA 1.7. If $a$ is an element of a semiprime commutative ring $A$, then ann $a=\bigcap\left\{Q: Q \in \mathscr{P}_{a}\right\}$.

We now have at hand all the tools with which to prove Theorem 1.

Proof of necessity. If $A$ is a Baer ring, then by Lemma 1.6 and Proposition 1.4, the mapping $Q \rightarrow Q \cap E$ is a bijection of $\mathscr{P}(A)$ upon $\mathscr{P}(E)$. By the remark following that proposition, the inverse of this bijection is $P \rightarrow \bar{P}$. The space $\mathscr{P}(E)$ is compact, and the space $\mathscr{P}(A)$ is Hausdorff, so to complete the proof, we need only show that the mapping $P \rightarrow \bar{P}$ is continuous.

Hence, let $a$ be an element of $A$. We must show that $\{P \in \mathscr{P}(E)$ : $a \notin \bar{P}\}$ is open in $\mathscr{P}(E)$. Let $e$ be the idempotent for which ann $a=$ Ae. By Lemma 1.5, $a \notin \bar{P}$ if and only if ann $a \subset \bar{P}$, and so $a \notin \bar{P}$ if and only if $e \in \bar{P}$, i.e., if and only if $e \in P$. We have shown that $\{P: a \notin \bar{P}\}=\{P: e \in P\}$. The latter set is both open and closed in $\mathscr{P}(E)$, and so the mapping $P \rightarrow \bar{P}$ is continuous.

Proof of sufficiency. For $a \in A$, the set $\mathscr{P}_{a}$ is both open and closed in $\mathscr{P}(A)$. Since the mapping $Q \rightarrow Q \cap E$ is a homeomorphism, the set $\{Q \cap E: a \notin Q\}$ is both open and closed in $\mathscr{P}(E)$. Hence, there is an idempotent $e$ in $E$ such that $\{Q \cap E: a \notin Q\}=\{Q \cap E: e \notin Q \cap E\}$. The latter set is the same as $\{Q \cap E: e \notin Q\}$, and consequently, $\{Q \in \mathscr{P}(A)$ : $a \notin Q\}=\{Q \in \mathscr{P}(A): e \notin Q\}$. This equality and Lemma 1.7 imply that 
ann $a=$ anne. But ann $e=A e^{\prime}$, and hence $A$ is a Baer ring.

As the following discussion will show, there are rings $A$ for which the mapping $Q \rightarrow Q \cap E$ is a bijection of $\mathscr{P}(A)$ upon $\mathscr{P}(E)$, but which are not Baer rings.

A semiprime ring $A$ is called complementedly normal [2, p. 196] if whenever $a, b$ are elements of $A$ such that $a b=0$ then there is an idempotent $e$ in $A$ such that $a e=0=b e^{\prime}$.

Now the ring $C(X)$ of real-valued continuous functions on a completely regular Hausdorff space $X$ is complementedly normal if and only if $X$ is a $U$-space [2, p. 218], and it is a Baer ring if and only if $X$ is basically disconnected, i.e., if and only if the lattice $C(X)$ is conditionally $\sigma$-complete $[9$, p. 45]. There are $U$-spaces which are not basically disconnected spaces [4, p. 390], so in virtue of Proposition 1.4 and the following result, there are semiprime rings $A$ which are not Baer rings, but for which the mapping $Q \rightarrow Q \cap E$ is a bijection of $\mathscr{P}(A)$ upon $\mathscr{P}(E)$.

LEMMA 1.8. If the semiprime ring $A$ is complementedly normal, then each minimal prime ideal in $A$ is regular.

Proof. Let $Q$ be a minimal prime ideal in the complementedly normal ring $A$. If $a$ is an element of $Q$, then by Lemma 1.5, there is an element $b \notin Q$ such that $a b=0$. Hence, there is an idempotent $e$ such that $a e^{\prime}=0=b e$. We must have $e$ in $Q$, and so $a$ is in $\bar{Q}$. Thus, each minimal prime ideal is regular.

2. Retracts. Recall that Spec $A$, the prime spectrum of a commutative ring $A$, is the space of all prime ideals of $A$. This section will be devoted to a proof of the following result.

THEOREM 2. A semiprime commutative ring $A$ with identity is a Baer ring if and only if the minimal prime spectrum of $A$ is a retract of the prime spectrum of $A$.

The above result was suggested by a paper of DeMarco and Orsatti [3], and some of the arguments used in its proof are similar to arguments used by those authors.

For $P$ in Spec $A$, let $O_{P}$ be the intersection of all minimal prime ideals contained in $P$. A proof of the following result can be found in $[2$, p. 105] and [3, p. 460].

LEMMA 2.1. In a semiprime commutative ring $A, O_{P}=\{a \in A$ : ann $a \not \subset P$ f for each prime ideal $P$. 
For a subset $S$ of $A$, let $h(S)$ be the set of all prime ideals which contain $S$, and for a subset $S$ of Spec $A$, let $k(S)$ be the intersection of all members of $S$. It is well-known and easy to prove that closed subsets of Spec $A$ are exactly those of the form $h(S)$ for some subset $S$ of $A$, and that the closure of a subset $S$ of Spec $A$ is $h(k(S))$. In particular then, the closure of a point $P$ of Spec $A$ consists of all prime ideals which contain $P$.

The next result is essentially Lemma 9.2 of [9].

Lemma 2.2. An ideal $I$ in a commutative ring $A$ with identity is a direct summand of $A$ if and only if $h(I)$ is an open-as well as closed-subset of Spec $A$.

We now prove the necessity of Theorem 2. If $a$ is an element of any commutative ring $A$, then ann $a \cap$ ann ann $a$ is contained in each prime ideal of $A$. This remark and Lemma 2.1 imply that $O_{P} \subset\{a \in$ $A$ : ann ann $a \subset P$ \} for each prime ideal $P$ in a semiprime ring $A$. A Baer ring $A$ is semiprime, and ann $a+$ ann ann $a=A$ for each element $a$ in such a ring, so $O_{P}=\{a \in A$ : ann ann $a \subset P\}$. In a semiprime ring, ann $a n n a b=a n n a n n a \cap a n n a n n b$ for each pair $a, b$. Therefore, $O_{P}$ is a prime ideal for each prime ideal $P$ in a Baer ring.

Now $\left\{P \in \operatorname{Spec} A: a \notin O_{P}\right\}=h(\operatorname{ann} a)$, and, by Lemma 2.2, in a Baer ring $A$, the latter set is open in $\operatorname{Spec} A$, so $P \rightarrow O_{P}$ is a continuous mapping of Spec $A$ into $\operatorname{Min} \operatorname{Spec} A$.

In any ring, obviously $a \in$ ann ann $a$, and thus $O_{P} \subset P$. Hence, in a Baer ring, $O_{P}=P$ for each minimal prime ideal $P$.

We have shown that the mapping $P \rightarrow O_{P}$ is a retraction of $\operatorname{Spec} A$ upon Min Spec $A$ when $A$ is a Baer ring.

The sufficiency of the condition in Theorem 2 for a ring to be a Baer ring is included in the following result. In proving this result, we use the easily verified fact that a semiprime commutative ring $A$ with identity is a Baer ring if ann ann a direct summand of $A$ for each element $a$.

Proposition 2.3. Let $A$ be a semiprime commutative ring with identity. If $\tau$ is a retraction of $\operatorname{Spec} A$ upon Min Spec $A$, then $\tau(P)=$ $O_{P}$ for each $P$ in Spec $A$, and $A$ is a Baer ring.

Proof. If $Q \in \mathscr{P}(A)$, then $Q=\tau(Q)$, i.e., $Q \in \tau^{\leftarrow}(Q)$. Since $\tau$ is a continuous mapping, and since $\mathscr{P}(A)$ is a Hausdorff space, $\tau^{\leftarrow}(Q)$ is closed in $\operatorname{Spec} A$, so $\mathrm{cl}\{Q\} \subseteq \tau^{\leftarrow}(Q)$. Thus, if $P$ is in Spec $A$, and $P \supset Q$, then $\tau(P)=Q$. Consequently, each prime ideal in $A$ contains 
a unique minimal prime ideal, and so $\tau(P)=O_{P}$ for each $P$ in $\operatorname{Spec} A$.

Since $A$ is semiprime, Lemma 2.1 insures that $\left\{P \in \operatorname{Spec} A: a \notin O_{P}\right\}=$ $h($ ann $a)$ for each element $a$ in $A$. Since the mapping $P \rightarrow O_{P}$ is continuous, h(ann $a)$ is open as well as closed for each $a$. By Lemma 2.2, ann $a$ is a direct summand of $A$ for each $a$, and thus $A$ is a Baer ring.

3. von Neumann regular rings. In this section, we shall apply Theorems 1 and 2 to obtain a new proof of the following result.

THEOREM 3.1. Let $A$ be a semiprime commutative ring with identity. If each prime ideal in $A$ is maximal, then $A$ is a von Neumann regular ring.

REMARK. As is well-known, the above theorem has a valid converse: if $A$ is a commutative von Neumann regular ring, then $A$ is semiprime-in fact, even semisimple-and each prime ideal in $A$ is maximal.

Proofs of Theorem 3.1 have been given by Cornish [2] and Peercy [11]. In his book on commutative rings [7], Kaplansky leaves the proof as an exercise, but with hints for doing it; Cornish's proof is essentially the one outlined by Kaplansky. Peercy's proof is a sheaftheoretic one, while the other two are not. Although we also use some results from sheaf theory to prove Theorem 3.1, our proof is different from Peercy's.

We begin with a summary of Pierce's [12] representation of a commutative ring with identity.

Recall that a sheaf $(\mathscr{B}, Y)$ of commutative rings is reduced if (i) $Y$ is a Boolean space, i.e., a compact Hausdorff space with a base of open-and-closed sets, and (ii) for each $y \in Y$ the only idempotents in the stalk $\mathscr{B}_{y}$ are $0_{y}$ and $1_{y}$.

THEOREM 3.2. (Pierce) Let $A$ be a commutative ring with identity, and for each $P \in \mathscr{P}(E)$, let $\mathscr{A}_{P}=(A / \bar{P}, P)$. Then $\mathscr{A}=\bigcup\left\{\mathscr{A}_{P}: P \in\right.$ $\mathscr{P}(E)\}$ is the sheaf space of a sheaf of reduced commutative rings with base space $\mathscr{P}(E)$, and the mapping $a \rightarrow \hat{a}$, where $\hat{a}(P)=a / \bar{P}$ is an isomorphism of $A$ upon the ring $\Gamma(\mathscr{P}(E), \mathscr{A})$ of global sections of the sheaf $(\mathscr{A}, \mathscr{P}(E))$.

For the remainder of this section, $(\mathscr{A}, \mathscr{P}(E))$ will denote the sheaf of reduced commutative rings defined above.

The following result is contained in Pierce's memoir [12]; it should 
be noted that the proof of its necessity is essentially a proof of the assertion that every prime ideal in a commutative von Neumann regular ring is maximal.

Theorem 3.3. A commutative ring $A$ with identity is a von Neumann regular ring if and only if each stall $(A / \bar{P}, P)$ of the sheaf $(\mathscr{A}, \mathscr{P}(E))$ is a field.

We are now ready to prove Theorem 3.1. To do so, let each prime ideal in the semiprime ring $A$ be maximal. Thus, $\mathscr{P}(A)=$ Spec $A$, and so, trivially, the first of these spaces is a retract of the second. By Theorem 2, $A$ is a Baer ring. Theorem 1 can obviously be recast as follows: A semiprime commutative ring $A$ with identity is a Baer ring if and only if the mapping $P \rightarrow \bar{P}$ is a homeomorphism of $\mathscr{P}(E)$ with $\mathscr{P}(A)$. Therefore, $\bar{P}$ is a prime ideal in $A$ for each $P \in \mathscr{P}(E)$, and thus it is maximal. By Theorem 3.3 , the ring $A$ is von Neumann regular.

4. Another application of Theorem 2. It is known [5] that every prime ideal in the ring $C(X)$ of all real-valued continuous functions on a completely regular space $X$ is contained in a unique maximal ideal. For each prime ideal $P$ in $C(X)$, let $\mu(P)$ be the unique maximal ideal containing it, and let $\iota$ be the restriction of $\mu$ to $\mathscr{P}(C(X))$, the space of minimal prime ideals in $C(X)$. The following result was obtained by Henriksen and Jerison [6].

THEOREM 4.1. (a) $c$ is a continuous mapping of $\mathscr{P}(C(X))$ onto $\beta X$, the Stone-Cech compactification of $X$.

(b) \& maps no proper closed subset of $\mathscr{P}$ onto $\beta X$.

(c) $c$ is one-to-one if and only if each prime ideal contains a unique minimal prime ideal, i.e., $X$ is an F-space.

(d) $C$ is a homeomorphism if and only if $X$ is basically disconnected.

(e) If $X$ is an $F$-space, then $\mathscr{P}(C(X))$ is compact if and only if $X$ is basically connected.

Now let $A$ be a commutative ring with identity in which each prime ideal is contained in a unique maximal ideal. For $P$ in $\operatorname{Spec} A$, let $\mu(P)$ be the unique maximal ideal containing $P$, and let $\iota$ be the restriction of $\mu$ to $\mathscr{P}(A)$. Let $\mathscr{C}(A)$ be the space of maximal ideals of $A$. In case $A=C(X), \mathscr{C}(A)$ is homeomorphic with $\beta X$, so the following theorem is a generalization of the above result of Henriksen and Jerison. 
THEOREM 4.2. (a) $c$ is a continuous mapping of $\mathscr{P}(A)$ upon $\mathscr{M}(A)$.

(b) If $A$ is semisimple, that is, if 0 is the only element common to all maximal ideals in $A$, then 6 maps no proper closed subset of $\mathscr{P}$ upon $\mathscr{A l}$.

(c) $\iota$ is injective if and only if each prime ideal in $A$ contains a unique minimal prime ideal.

(d) If $A$ is semiprime, then 6 is a homeomorphism if and only if $A$ is a Baer ring.

(e) If each prime ideal in the semiprime ring $A$ contains a unique minimal prime ideal, then $\mathscr{P}(A)$ is compact if and only if $A$ is a Baer ring.

Proof. (a) This is a consequence of the fact, established by DeMarco and Orsatti [3], that $\mu$ is a continuous mapping of $\operatorname{Spec} A$ upon $\mathscr{C}(A)$.

(b) This can be proved in the same way that Henriksen and Jerison proved (b) of Theorem 4.1. We repeat their argument. Every proper closed set in $\mathscr{P}$ is contained in a set of the form $h(a)$ for some nonzero element $a$ in $A$, because such sets form a base for the closed sets. If $M$ is a maximal ideal such that $a \notin M$, then $M \notin \iota(h(a))$.

(c) It is easy to see that the following three statements are equivalent:

(i) $c$ is one-to-one;

(ii) each maximal ideal contains a unique minimal prime ideal;

(iii) $O_{M}$ is a minimal prime ideal for each maximal ideal $M$.

For each prime ideal $P$ in $A$, we have $O_{\mu(P)} \subset P \subset \mu(P)$. Thus, if (iii) holds, then each prime ideal contains a unique minimal prime ideal. Conversely, if each prime ideal contains a unique minimal prime ideal, then, in particular, $O_{M} \in \mathscr{P}(A)$ for each $M$ in $\mathscr{M}(A)$, so $c$ is one-to-one with inverse $M \rightarrow O_{M}$.

(d) If $A$ is a Baer ring, then by Theorem 2, the mapping $P \rightarrow O_{P}$ is a retraction of $\operatorname{Spec} A$ upon $\mathscr{P}(A)$. In particular then, the continuous mapping $M \rightarrow O_{M}$ of $\mathscr{C}(A)$ upon $\mathscr{P}(A)$ is the inverse of $\iota$, so the latter mapping is a homeomorphism. Conversely, if $\iota$ is a homeomorphism, then the composition $P \rightarrow \mu(P) \stackrel{c^{-1}}{\longrightarrow} O_{\mu(P)}=O_{P}$ is a retraction of Spec $A$ upon $\mathscr{P}(A)$, so by Theorem 2, the semiprime ring $A$ is a Baer ring.

(e) is a consequence of (c), (d), and the fact, again established by DeMarco and Orsatti [loc. cit.], that $\mathscr{M}(A)$ is a Hausdorff space.

\section{REFERENCES}

1. W. H. Cornish, The variety of commutative Rickart rings, to appear in Nanta Mathematica. 
2. Abelian Rickart semirings, Thesis, School of Mathematical Sciences, The Flinders University of South Australia, 1970.

3. Giuseppe DeMarco and Adelberto Orsatti, Commutative rings in which every prime ideal is contained in a unique maximal ideal, Proc. Amer. Math. Soc., 30 (1971), 459466.

4. L. Gillman and M. Henriksen, Rings of continuous functions in which every finitely generated ideal is principal, Trans. Amer. Math. Soc., 82 (1956), 366-391.

5. L. Gillman and M. Jerison, Rings of Continuous Functions, Van Nostrand, Princeton, N. J., 1960.

6. M. Henriksen and M. Jerison, The space of minimal prime ideals of a commutative ring, Trans. Amer. Math. Soc., 115 (1965), 110-130.

7. Irving Kaplansky, Commutative Rings, Allyn and Bacon, Inc., Boston, 1970.

8. Klaus Keimel, Baer extensions of rings and Stone extensions of semigroups, Semigroup Forum, 2 (1971), 55-63.

9. J. Kist, Minimal prime ideals in commutative semigroups, Proc. London Math. Soc., (3) 13 (1963), 31-50.

10. Compact spaces of minimal prime ideals, Math. Z., 111 (1969), 151-158. 11. D. E. Peercy, The complete Baer extension of a commutative ring, Thesis, New Mexico State University, 1970.

12. R. S. Pierce, Modules over commutative regular rings, Memoirs Amer. Math. Soc., No. 70 (1967).

13. D. Scott, On Constructing Models for Arithmetic, Infinitistic Methods, Proceedings of the symposium on Foundations of Mathematics, 235-255, Pergamon Press, 1961.

14. T. P. Speed and M. W. Evans, A note on commutative Baer rings, J. Austral. Math. Soc., 13 (1972), 1-6.

15. — A note on commutative Baer rings-II, to appear in J. Australian Math. Soc.

16. - A note on commutative Baer rings III, to appear in J. Australian Math. Soc.

17. - On commutative Baer rings, to appear.

Received September 29, 1972.

New Mexico State University 


\section{PACIFIC JOURNAL OF MATHEMATICS}

\section{EDITORS}

RICHARD ARENS (Managing Editor) University of California

Los Angeles, California 90024

R. A. BeaUmont

University of Washington Seattle, Washington 98105
J. DUGUNDJI*

Department of Mathematics University of Southern California Los Angeles, California 90007

D. Gilbarg and J. Milgram Stanford University

Stanford, California 94305

\section{ASSOCIATE EDITORS}

E. F. BECKENBACH

B. H. NeUmanN

F. WOLF

K. YoSHIDA

\section{SUPPORTING INSTITUTIONS}

\section{UNIVERSITY OF BRITISH COLUMBIA CALIFORNIA INSTITUTE OF TECHNOLOGY UNIVERSITY OF CA.LIFORNIA MONTANA STATE UNIVERSITY UNIVERSITY OF NEVADA NEW MEXICO STATE UNIVERSITY OREGON STATE UNIVERSITY UNIVERSITY OF OREGON OSAKA UNIVERSITY}

UNIVERSITY OF SOUTHERN CALIFORNIA STANFORD UNIVERSITY UNIVERSITY OF TOKYO UNIVERSITY OF UTAH WASHINGTON STATE UNIVERSITY UNIVERSITY OF WASHINGTON AMERICAN MATHEMATICAL SOCIETY NAVAL WEAPONS CENTER

* C. R. DePrima California Institute of Technology, Pasadena, CA 91109, will replace J. Dugundji until August 1974. 


\section{Pacific Journal of Mathematics}

\section{Vol. 50, No. $1 \quad$ September, 1974}

Gail Atneosen, Sierpinski curves in finite 2-complexes.............. 1

Bruce Alan Barnes, Representations of $B^{*}$-algebras on Banach spaces .... 7

George Benke, On the hypergroup structure of central $\Lambda(p)$ sets ....... 19

Carlos R. Borges, Absolute extensor spaces: a correction and an

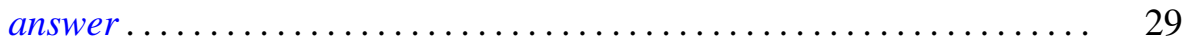

Tim G. Brook, Local limits and tripleability .................. 31

Philip Throop Church and James Timourian, Real analytic open maps .... 37

Timothy V. Fossum, The center of a simple algebra ............... 43

Richard Freiman, Homeomorphisms of long circles without periodic

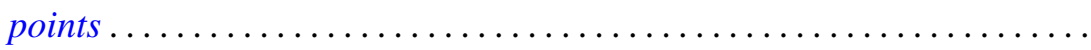

B. E. Fullbright, Intersectional properties of certain families of compact

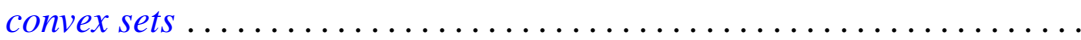

Harvey Charles Greenwald, Lipschitz spaces on the surface of the unit

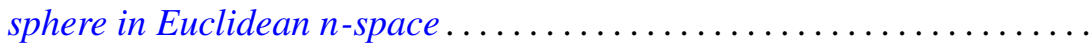

Herbert Paul Halpern, Open projections and Borel structures for

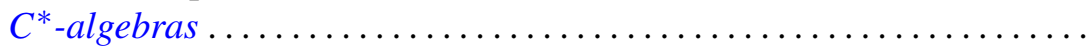

Frederic Timothy Howard, The numer of multinomial coefficients divisible

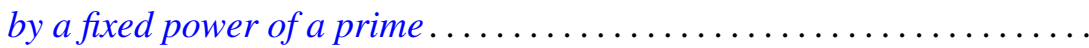

Lawrence Stanislaus Husch, Jr. and Ping-Fun Lam, Homeomorphisms of manifolds with zero-dimensional sets of nonwandering points........ 109

Joseph Edmund Kist, Two characterizations of commutative Baer rings ...

Lynn McLinden, An extension of Fenchel's duality theorem to saddle functions and dual minimax problems ................

Leo Sario and Cecilia Wang, Counterexamples in the biharmonic classification of Riemannian 2-manifolds...

Saharon Shelah, The Hanf number of omitting complete types ...

Richard Staum, The algebra of bounded continuous functions into a

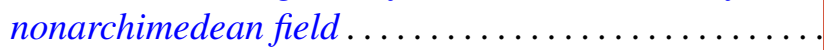

James DeWitt Stein, Some aspects of automatic continuity ..

Tommy Kay Teague, On the Engel margin

John Griggs Thompson, Nonsolvable finite groups all of whose local subgroups are solvable, $V \ldots \ldots \ldots \ldots \ldots \ldots \ldots \ldots$

Kung-Wei Yang, Isomorphisms of group extensions 